\title{
高分散镍-硼/纳米多孔铜非晶态合金电极上葡萄糖的电催化氧化
}

\author{
张树金郑一雄* 袁林珊 杨卫华 \\ (华侨大学材料科学与工程学院 厦门 361021)
}

\begin{abstract}
摘要 通过酸腐蚀去合金法处理热扩散制备的铜基表面铜锌合金得到纳米多孔铜(NPC)材料. 以 NPC 为载体, 采用超 声辅助化学镀制备 Ni-B/NPC 合金电极. X射线衍射(XRD)和扫描电镜(SEM) 表明 Ni-B/NPC 电极呈现由高分散纳米颗粒 组成的非晶态结构. 计时电流法 $(\mathrm{CA})$ 结果表明化学镀 $5 \mathrm{~min}$ 制备的 Ni-B/NPC 电极具有最大的电化学活性表面积 (EASA). 循环伏安法 $(\mathrm{CV})$ 结果表明, 与块状镍电极相比, 碱性介质中在 Ni-B/NPC 电极上葡萄糖起始氧化电位负移 39 $\mathrm{mV}$, 氧化峰电流提高了 18.9 倍. 采用线性扫描伏安法(LSV)、CA 和电化学阻抗谱(EIS)测定 Ni-B/NPC 电极对葡萄糖电 催化氧化的电子转移系数 $(\beta)$ 、电催化氧化反应速率常数 $(k)$ 和葡萄糖的扩散系数 $(D)$ 等动力学参数. 结果表明高分散 $\mathrm{Ni}-\mathrm{B} / \mathrm{NPC}$ 非晶态合金电极对碱性介质中葡萄糖的氧化具有较高的电催化活性和稳定性.

关键词 纳米多孔铜; 超声辅助化学镀; 镍-嗍非晶态合金; 葡萄糖; 电催化氧化
\end{abstract}

\section{Electrocatalytic Oxidation of Glucose on Highly Dispersed Ni-B/Nanoporous Cu Amorphous Alloy Electrode}

\author{
Zhang, Shujin Zheng, Yixiong* Yuan, Linshan Yang, Weihua \\ ( College of Materials Science and Engineering, Huaqiao University, Xiamen 361021)
}

\begin{abstract}
As glucose is the richest carbohydrate in nature and has extremely high energy density, direct glucose fuel cell (DGFC) is considered to be a promising power source. Catalyst is the key to the development of DGFC. The expensive noble metal catalysts hinder their commercial applications. Ni is a cheap metal with an excellent catalytic performance. Ni and its compounds using glassy carbon as the carrier have been developed for glucose oxidation, but the smooth surface of glassy carbon could not be conductive to dispersion of nanoparticles. The development of nanoporous materials may overcome the drawback. In this experiment, $\mathrm{Cu}$-based surface $\mathrm{Cu}-\mathrm{Zn}$ alloy was prepared by thermal diffusion and Nanoporous Copper (NPC) with high surface area was prepared by removing of $\mathrm{Zn}$ element from the alloy in $5 \% \mathrm{H}_{2} \mathrm{SO}_{4}$ solution. NPC supported Nickel-Boron (Ni-B/NPC) alloy electrode was fabricated by ultrasonic-assisted electroless plating. The Ni-B/NPC alloy electrode was characterized by scanning electron microscopy (SEM), X-ray diffraction (XRD) and electrochemical methods. XRD and SEM results showed Ni-B alloy electrode was amorphous structure with high dispersion of Ni-B nanoparticles. Chronoamperometry (CA) result found the prepared Ni-B/NPC amorphous alloy electrode with ultrasonic for 5 min had the highest electrochemical active surface area (EASA) and surface roughness. The CV results of glucose oxidation at the $\mathrm{Ni}-\mathrm{B} / \mathrm{NPC}$ amorphous alloy electrode showed the onset oxidation potential had a negative shift of $39 \mathrm{mV}$ and the oxidation peak current density increased by 18.9 times compared with the bulk Ni electrode in alkaline media. Meanwhile, the kinetic parameters of electrocatalytic oxidation of glucose at the Ni-B/NPC amorphous alloy electrode were determined by linear sweep voltammetry (LSV), CA method and electrochemical impedance spectroscopy (EIS), such as charge transfer coefficient $(\beta)$, catalytic rate constant $(k)$ and diffusion coefficient $(D)$. The results indicated the good electrocatalytic activity and stability of the highly dispersed Ni-B/NPC amorphous alloy electrode for glucose oxidation in alkaline medium, and the diffusion coefficient of glucose was improved by about two orders of magnitude relative to the reported value at the $\mathrm{Ni}-\mathrm{Cu} / \mathrm{GC}$ electrode. The Ni-B alloy should be a promising anodic catalyst for direct glucose fuel cell.
\end{abstract}

Keywords nanoporous copper; ultrasonic-assisted electroless; nickel-boron amorphous alloy; glucose; electrocatalytic oxidation

\section{1 引言}

随着化石燃料日益枯竭, 寻找可再生能源是科学研
究者亟待解决的重大问题. 在过去的几十年, 燃料电池 作为一种高效、清洁和环保的能源技术得到迅速的发展. 其中，直接利用取自有机物的生物质然料电池越来越受

* E-mail: yixiongzheng@126.com; Tel.: 0086-0595-22692512

Received May 31, 2013; published November 3, 2013.

Project supported by the Fujian Provincial Natural Science Foundation (No. 2010J01292), Fund of Fujian Provincial Key Laboratory of Nanomaterials (No. NM10-04), and Program for Excellent Talents in Huaqiao University, China (No. 08BS205), and the National Natural Science Foundation of China (No. 21103055).

项目受福建省自然科学基金(No. 2010J01292)、福建省纳米材料重点实验室基金(No. NM10-04)、华侨大学高层次人才科研启动基金(No. 08BS205)和 国家自然科学基金(No. 21103055)资助. 
到人们的注意. 在自然界中, 葡萄糖是最丰富的碳水化 合物, 且具有很高的能量密度, 通过转移 24 个电子完全 氧化成 $\mathrm{CO}_{2}$ 可以产生 $2870 \mathrm{~kJ} \bullet \mathrm{mol}^{-1}$ 的能量. 利用葡萄糖 无需经过发酵或重整制取的特点可以降低成本的独特 优势, 直接葡萄糖燃料电池(DGFC)越来越引起人们的 重视 ${ }^{[1 \sim 3]}$.

尽管微生物和酶葡萄糖燃料电池取得了一定的发 展, 但是较低的电流和能量密度、高昂的维护费用、电 极上生物催化剂固定困难、寿命短及稳定性差等缺点阻 碍了该类燃料电池的发展 ${ }^{[3 \sim 5]}$. 为了克服微生物和酶葡 萄糖燃料电池的缺点, 由金属催化剂构筑的无酶电极吸 引了人们的目光. 在过去的几年中, 用于葡萄糖电催化 氧化的铂 ${ }^{[6]}$ 、钯 ${ }^{[7]}$ 和金 ${ }^{[8]}$ 及其合金 ${ }^{[9 \sim 11]}$ 等贵金属电极相继 研究出来，但其价格昂贵从而限制了商业化应用.

由于金属镍是一种来源广、价格低廉和电催化性能 良好的金属. 近年来, 关于镍及其化合物电极用于葡萄 糖电催化氧化成为人们研究的重点领域. 如 Yousef Elahi 等 ${ }^{[12]}$ 用姜黄素改性镍玻碳电极; Qiu 等 ${ }^{[13]}$ 制备出树 枝状铜镍合金电极; Jafarian 等 ${ }^{[14]}$ 在玻碳电极上恒电流 沉积镍和铜镍合金电极; Zheng 等 ${ }^{[15]}$ 制备了镍离子和槲 皮素复合改性碳纳米管离子液体糊状电极; Ganesh 等 ${ }^{[16]}$ 在 ITO 电极上沉积氢氧化镍; Chekin 等 ${ }^{[17]}$ 合成了镍/聚 丙烯腈和碳纳米管复合改性电极; Chen 等 ${ }^{[18]}$ 在泡沫镍 上沉积银纳米粒子制备了泡沫银/镍电极; Galindo 等 ${ }^{[19]}$ 用电化学方法合成铁酸镍电极; Danaee 等 ${ }^{[20]}$ 通过恒电流 法沉积镍修饰玻碳电极; El-Refaei 等 ${ }^{[21]}$ 采用锰镍的复 合氧化物改性玻碳电极. 尽管这些镍基催化剂对葡萄糖 的电催化氧化呈现一定的活性和抗毒化能力, 但低表面 积的载体限制其电催化活性. 同时, 纳米颗粒与光滑的 玻碳载体作用力不强, 在长时间的测试条件下会影响电 极的稳定性和寿命. 去合金法 ${ }^{[22]}$ 制备的纳米多孔金属 材料具有极大的比表面积、较高机械强度、良好的导电 性和抗腐蚀能力, 是理想的电催化剂载体材料.

经研究发现镍基非晶态合金是优良的催化加氢材 料 ${ }^{[23]}$, 也是性能优越的催化析氢阴极材料 ${ }^{[24]}$, 但它们作 为葡萄糖阳极电催化剂的应用报道非常少.

近几年, 我们实验室一直从事镍基非晶态合金作为 阳极电催化剂用于醇类小分子的电催化氧化的研 究 ${ }^{[25,26]}$. 由于传统的多孔金和多孔银成本较高, 本文通 过热扩散法制备铜基表面铜锌合金，再结合酸腐蚀去合 金法 ${ }^{[27]}$ 得到纳米多孔铜载体, 采用超声波辅助化学镀 成功制备出高分散 Ni-B/NPC 非晶态合金电极; 最后, 研究了该电极对葡萄糖的电催化氧化行为.

\section{2 结果与讨论}

\section{1 纳米多孔铜及高分散 Ni-B/NPC 非晶态合金电极 的形貌及结构}

图 1 为热处理前后 $\mathrm{Cu}-\mathrm{Zn}$ 样品、纳米多孔铜及超声
辅助化学镀 Ni-B/NPC 非晶态合金电极的 XRD 图谱. 热 处理前的镀锌铜片 (图 1 曲线 a) 在 $39.1^{\circ}$ (100)和 $70.8^{\circ}$ (110)处呈现锌的衍射峰对应于 JCPDS: 04-0831, 在 $43.4^{\circ}(111), 50.5^{\circ}$ (200)和 $74.2^{\circ}$ (220) 处呈现铜的衍射 峰对应于 JCPDS: 04-0836. 在 $\mathrm{N}_{2}$ 保护下经过 $2 \mathrm{~h} 180{ }^{\circ} \mathrm{C}$ 热处理后, 锌和铜的衍射峰消失(图 1 曲线 b) 呈现 $\mathrm{CuZn}_{5}$ 合金衍射峰(JCPDS: 35-1151), 可见热处理可促使锌原 子和铜原子相互扩散而形成表面 $\mathrm{CuZn}_{5}$ 合金; 铜基表面 $\mathrm{CuZn}_{5}$ 合金在 $5 \% \mathrm{H}_{2} \mathrm{SO}_{4}$ 溶液中去合金化 $60 \mathrm{~min}$ 后，铜 的衍射峰重新出现, 合金的衍射峰消失(图 1 曲线 $\mathrm{c}$ ), 得 到纳米多孔铜; 超声辅助化学镀 Ni-B 合金后, 在 $2 \theta$ 为 $45^{\circ}$ 附近出现弥散峰(图 1 曲线 $\mathrm{f}$ ), 这是 $\mathrm{Ni}$ 系非晶态合金 的特征衍射峰 ${ }^{[26]}$ ，表明在纳米多孔铜表面形成了 $\mathrm{Ni}-\mathrm{B}$ 非晶态合金. 对比图 1 曲线 $\mathrm{d}$, e 和 $\mathrm{f}$, 可知镀液中 $\mathrm{KBH}_{4}$ 的浓度越高, 越有利于形成非晶态结构的 Ni-B 合金.

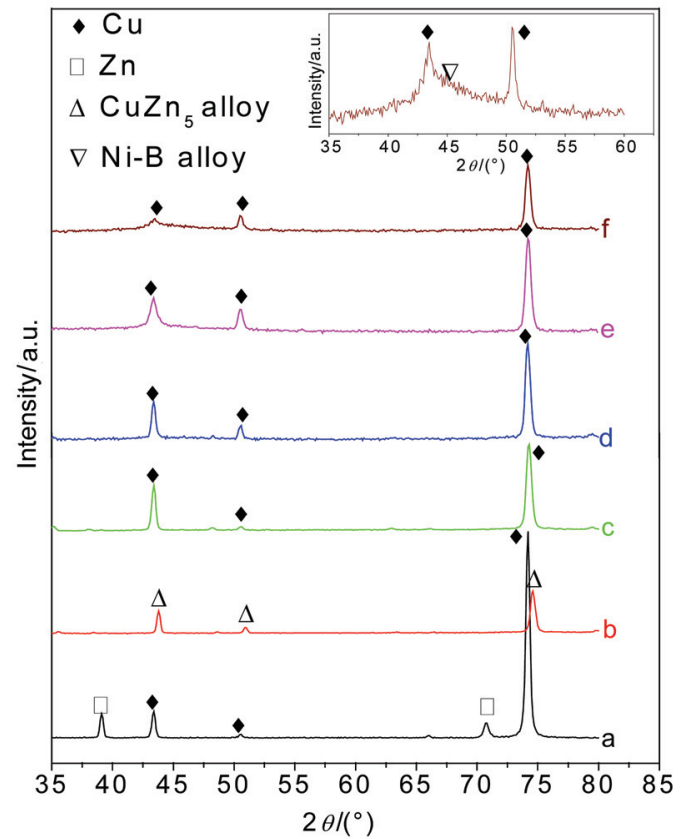

图 $1 \mathrm{Cu}-\mathrm{Zn}$ 样品的 XRD 图谱: (a)没有热处理; (b) $180{ }^{\circ} \mathrm{C}$ 热处理 $2 \mathrm{~h}$; (c)去合金化 $1 \mathrm{~h}$. 不同浓度 $\mathrm{KBH}_{4}$ 的镀液超声辅助化学镀 $\mathrm{Ni}-\mathrm{B}$ 非晶态 合金的 XRD 图谱: (d) $0.6 \mathrm{~g} \cdot \mathrm{L}^{-1}$; (e) $0.9 \mathrm{~g} \cdot \mathrm{L}^{-1}$; (f) $1.2 \mathrm{~g} \cdot \mathrm{L}^{-1}$, 内插图为 曲线 $\mathrm{f}$ 的局部放大图

Figure 1 X-ray diffraction patterns of $\mathrm{Cu}-\mathrm{Zn}$ samples: (a) without thermal treatment; (b) with thermal treatment at $180{ }^{\circ} \mathrm{C}$ for $2 \mathrm{~h}$; (c) dealloying for $1 \mathrm{~h}$. Electroless Ni-B amorphous alloy with ultrasonic in different concentrations of $\mathrm{KBH}_{4}$ solution: (d) $0.6 \mathrm{~g} \cdot \mathrm{L}^{-1}$; (e) $0.9 \mathrm{~g} \cdot \mathrm{L}^{-1}$; (f) $1.2 \mathrm{~g} \cdot \mathrm{L}^{-1}$. Inset: the partial magnified curve $\mathrm{f}$

图 2 为不同腐蚀时间制备得到的纳米多孔铜、无超 声辅助化学镀及超声辅助化学镀 $5 \mathrm{~min}$ 的 Ni-B/NPC 非 晶态合金电极电镜图. 正如图 $2 \mathrm{~A}$ 所示, 铜基表面 $\mathrm{CuZn}_{5}$ 合金经 5\% $\mathrm{H}_{2} \mathrm{SO}_{4}$ 去合金化 $20 \mathrm{~min}$ 后，呈现了纳米多孔 结构. 随着去合金化时间的增加，逐渐形成了双连续三 维纳米多孔结构. 去合金化 $60 \mathrm{~min}$ 后，纳米多孔结构的 骨架尺寸 100 200 nm，孔径尺寸约为 100～200 nm (图 2C). 正如图 2D 所示，随着去合金化时间的进一步增加， 
纳米多孔结构的骨架尺寸进一步变小, 但孔径尺寸分布 不均匀, 部分骨架破坏, 从而使得局部尺寸变大. 因此, 本文采用去合金化 $60 \mathrm{~min}$ 制备的纳米多孔铜作为载体. 图 2E 和图 $2 \mathrm{~F}$ 对比发现, 超声波的存在与否对 Ni-B 非 晶态合金纳米颗粒的分散性影响显著, 无超声辅助化学 镀 Ni-B 非晶态合金纳米颗粒的分散性较差, 纳米颗粒 团聚在一起, 而超声辅助化学镀 Ni-B/NPC 非晶态合金 表面形成均匀分布尺寸为 $150 \sim 300 \mathrm{~nm}$ 的纳米颗粒. 可 见, 超声辅助化学镀起到抑制 Ni-B 非晶态合金纳米颗 粒团聚的作用, 提高其分散性. 这是由于超声波具有良 好穿透能力, 使得镀液可以充分扩散到纳米多孔铜表 面，外加超声波的空化效应有利于抑制粒子的团聚，从 而制备出高分散的 Ni-B/NPC 非晶态合金电极, 大大提 高了电极的粗糙度.
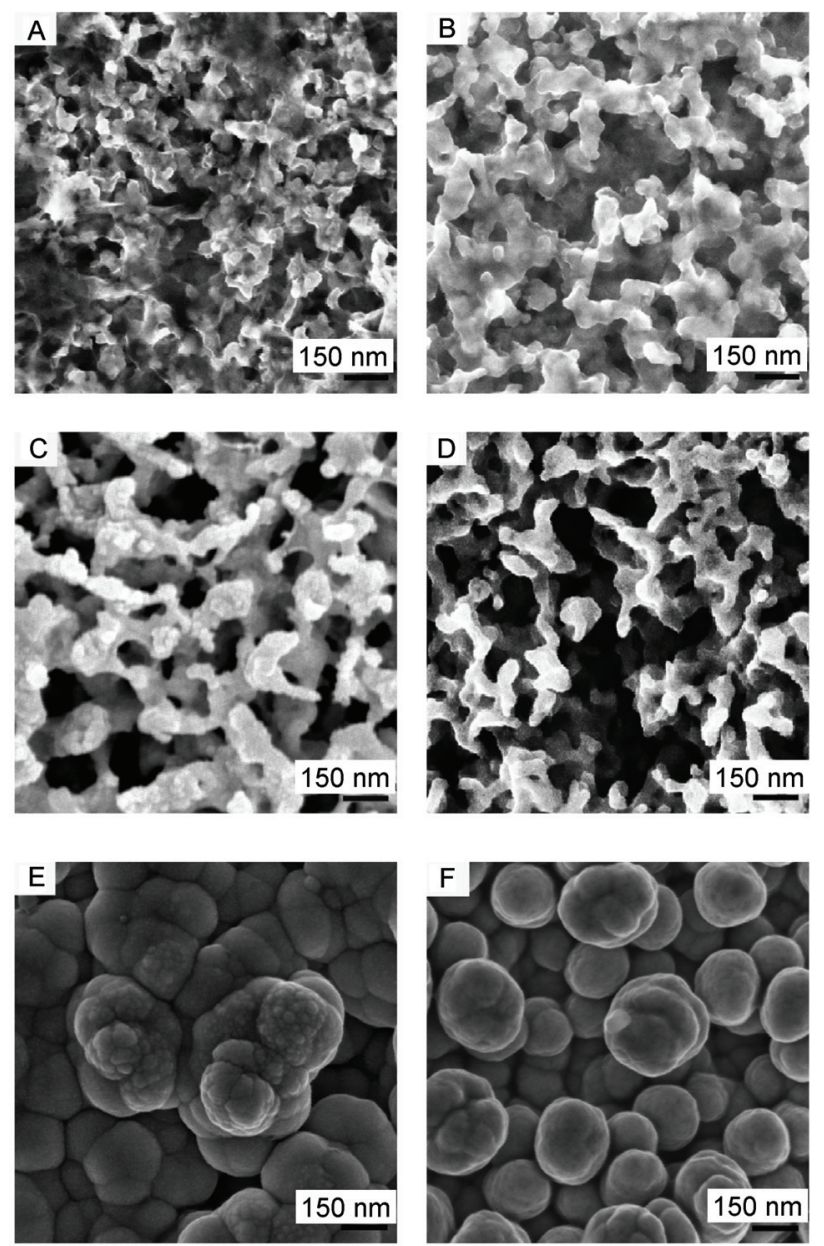

图 2 不同腐蚀时间制备得到的纳米多孔铜 $(\mathrm{A}) \sim(\mathrm{D})$ 、无超声 $(\mathrm{E})$ 与超 声(F)辅助化学镀 $5 \mathrm{~min}$ 的 Ni-B/NPC 非晶态合金电极的电镜照片

Figure 2 SEM images of $\mathrm{Cu}-\mathrm{Zn}$ alloy samples after dealloying for 20 $\min (A), 40 \min (B), 60 \min (C), 120 \min (D)$, electroless Ni-B/NPC amorphous alloy without ultrasonic (E) and with ultrasonic (F) for $5 \mathrm{~min}$

\section{2 高分散 Ni-B/NPC 非晶态合金电极电化学活性表 面积的测定}

图 3 为块状镍电极和不同超声辅助化学镀时间的高
分散 Ni-B/NPC 非晶态合金电极(A)和以光滑铜䈃(SC) 为基底, 不同超声辅助化学镀时间的 Ni-B/SC 非晶态合 金电极(B)在 $1 \mathrm{~mol} \cdot \mathrm{L}^{-1} \mathrm{NaOH}$ 溶液中的计时电流曲线. 通过微分电容法 ${ }^{[28]}$ 计算出电极微分电容 $\left(C_{\mathrm{d}}\right)$, 其值与单 位纯录电极表面的微分电容值 $\left(20 \mu \mathrm{F} \cdot \mathrm{cm}^{-2}\right)$ 对比, 可以 计算电极的 EASA，再利用电极的 EASA 除以表观面积 得到粗粘度. 其电化学活性表面积和粗糙度数据见表 1 . 显然超声辅助化学镀 $5 \mathrm{~min}$ 电极的 EASA 最大, 粗粘度 最高，说明高比表面积的载体有助于 Ni-B 非晶态合金 颗粒的分散，增加电极的 EASA. 镍电极表面的活性物 种 $\mathrm{NiOOH} / \mathrm{Ni}(\mathrm{OH})_{2}$ 相互转化过程的控制步骤是固相体 相中的质子扩散，电极具有很大的 EASA，可增加与电 解液的接触, 且减小质子在 $\mathrm{Ni}(\mathrm{OH})_{2}$ 固相中的扩散距 离, 提高质子的扩散性能 ${ }^{[29]}$, 从而提高电极的催化性 能. 因此, 后面研究高分散 Ni-B/NPC 非晶态合金电极 对葡萄糖电催化氧化时, 均采用超声辅助化学镀 $5 \mathrm{~min}$ 制备的电极.

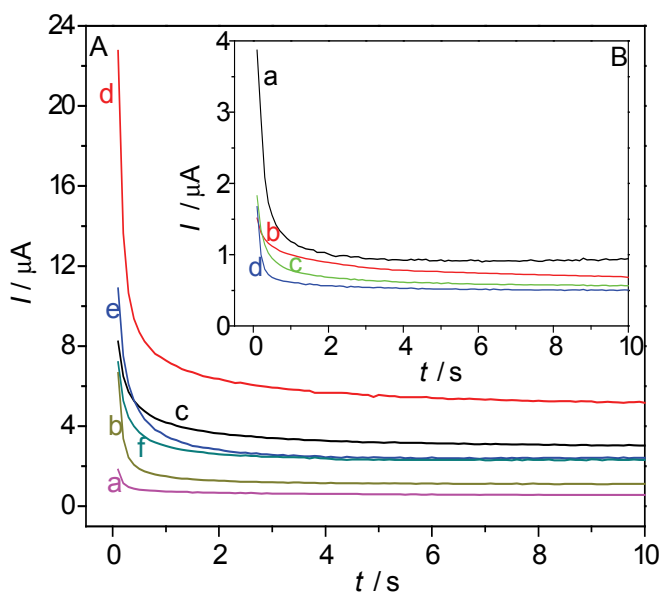

图 3 (A)块状镍电极(a), 无超声波化学镀 5 min (b)及超声波化学镀 3 $\min (\mathrm{c}), 5 \mathrm{~min}(\mathrm{~d}), 10 \mathrm{~min}(\mathrm{e}), 15 \mathrm{~min}(\mathrm{f})$ 制备的 Ni-B/NPC 非晶态合金 电极在 $1 \mathrm{~mol} \cdot \mathrm{L}^{-1} \mathrm{NaOH}$ 溶液中的计时电流曲线. (B)超声波化学镀 3 $\min (a), 5 \min (b), 10 \min (c), 15 \min$ (d)制备的 Ni-B/SC 非晶态合金电 极在 $1 \mathrm{~mol} \cdot \mathrm{L}^{-1} \mathrm{NaOH}$ 溶液中的计时电流曲线. 阶跃电势为 $0.1 \mathrm{~V}$ 到 $0.11 \mathrm{~V}$

Figure 3 (A) Chronoamperograms of the bulk nickel (a), Ni-B/NPC amorphous alloy electrodes without ultrasonic for $5 \mathrm{~min}$ (b) and with ultrasonic for $3 \mathrm{~min}$ (c), $5 \mathrm{~min}$ (d), $10 \mathrm{~min}$ (e), $15 \mathrm{~min}$ (f). (B) Chronoamperograms of Ni-B/SC amorphous alloy electrodes with ultrasonic for 3 $\min \left(\right.$ a), $5 \mathrm{~min}$ (b), $10 \mathrm{~min}$ (c), $15 \mathrm{~min}$ (d). Solution: $1 \mathrm{~mol} \cdot \mathrm{L}^{-1} \mathrm{NaOH}$; Step potential from $0.1 \mathrm{~V}$ to $0.11 \mathrm{~V}$

\section{3 高分散 Ni-B/NPC 非晶态合金电极在碱液中对葡 萄糖的电催化氧化活性}

图 4A 为高分散 Ni-B/NPC 非晶态合金电极在扫描 速率 $10 \mathrm{mV} \cdot \mathrm{s}^{-1}$ 含不同浓度葡萄糖的 $1 \mathrm{~mol} \cdot \mathrm{L}^{-1} \mathrm{NaOH}$ 溶 液中的循环伏安曲线. 与空白溶液的 $\mathrm{CV}$ 曲线 $\mathrm{a}$ 比较, 当溶液中加入葡萄糖时, $\mathrm{Ni}(\mathrm{OH})_{2}$ 的表观氧化峰电流密 度明显增大，且随着葡萄糖浓度的增加氧化峰电流也增 大. 这是由于正向扫描时，电极上的 $\mathrm{Ni}(\mathrm{OH})_{2}$ 氧化为 
表 1 不同电极的电化学活性表面积和粗粘度

Table 1 EASA and roughness of different electrodes with different electroless plating time

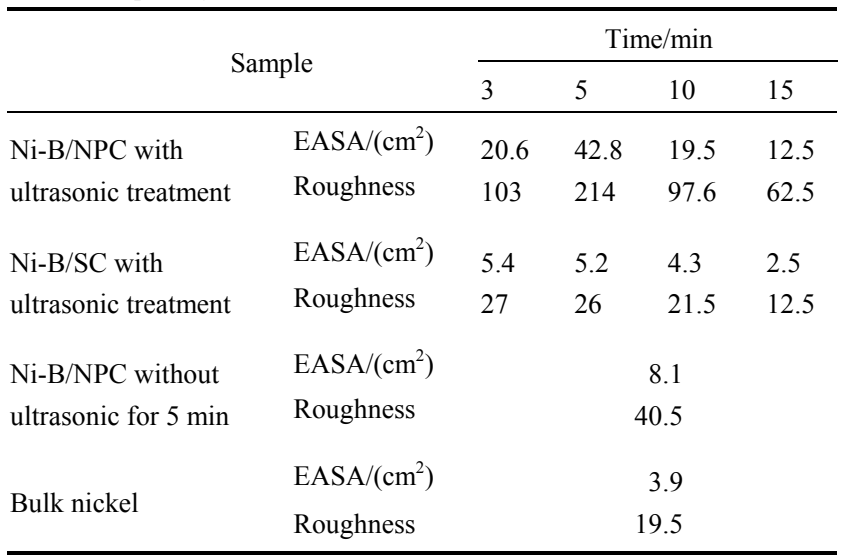

$\mathrm{NiOOH}, \mathrm{NiOOH}$ 是较强的氧化剂, 当葡萄糖扩散至电极 表面时, $\mathrm{NiOOH}$ 被葡萄糖还原为 $\mathrm{Ni}(\mathrm{OH})_{2}$. 葡萄糖浓度 越高, $\mathrm{NiOOH}$ 被葡萄糖还原为 $\mathrm{Ni}(\mathrm{OH})_{2}$ 的量越多, $\mathrm{Ni}(\mathrm{OH})_{2}$ 的氧化峰电流密度也越大. 说明电极表面吸附 的葡萄糖分子被氧化的同时, $\mathrm{Ni}(\mathrm{OH})_{2}$ 也被氧化为 $\mathrm{NiOOH}$. 随着扫描电势超过峰电势后, 氧化电流也逐渐 降低, 这可能是反应中间产物吸附在电极表面, 阻碍了 电极表面对葡萄糖分子的吸附. 电势反向扫描时, 前半 阶段的电流随着葡萄糖浓度的增大而增大, 说明正向扫 描电极表面未被氧化的中间产物在反向扫描的起始高 扫描电势下继续反应, 在电势反扫至 $0.3 \mathrm{~V}$ 时, 未被还 原的 $\mathrm{NiOOH}$ 再次被还原为 $\mathrm{Ni}(\mathrm{OH})_{2}$. 其中, 反扫过程中 出现两个还原峰是由 $\beta-\mathrm{NiOOH}$ 和 $\gamma-\mathrm{NiOOH}$ 分别形成 $\beta$ $\mathrm{Ni}(\mathrm{OH})_{2}$ 和 $\alpha-\mathrm{Ni}(\mathrm{OH})_{2}$ 所致 ${ }^{[30,31]}$. 由图 4B 可知, 阳极氧 化峰电流密度与葡萄糖浓度变化呈现线性关系.

图 5 为扫描速率 $10 \mathrm{mV} \cdot \mathrm{s}^{-1}$ 下块状镍电极和高分散 $\mathrm{Ni}-\mathrm{B} / \mathrm{NPC}$ 非晶态合金电极在含 $10 \mathrm{mmol} \cdot \mathrm{L}^{-1}$ 葡萄糖的 1 $\mathrm{mol} \cdot \mathrm{L}^{-1} \mathrm{NaOH}$ 溶液中的循环伏安曲线. 高分散 Ni-B/NPC 非晶态合金电极对葡萄糖的起始氧化电位为 $0.291 \mathrm{~V}$, 表观氧化峰电流密度为 $43.4 \mathrm{~mA} \cdot \mathrm{cm}^{-2}$, 而块状 镍电极对葡萄糖的起始氧化电位为 $0.330 \mathrm{~V}$, 表观氧化 峰电流密度为 $2.3 \mathrm{~mA} \cdot \mathrm{cm}^{-2}$. 显然高分散 $\mathrm{Ni}-\mathrm{B} / \mathrm{NPC}$ 非晶 态合金电极具有更高的葡萄糖电催化氧化活性. 其原因 可能是电极具有很高的粗楉度, 可大大增加与电解液的 接触, 使得表面吸附更多的葡萄糖分子, 从而电势正向 扫描时形成较大的氧化电流.

在研究催化剂的电化学性能时, 研究不同电势扫描 速率对电极反应的影响是很重要的. 图 $6 \mathrm{~A}$ 为高分散 $\mathrm{Ni}-\mathrm{B} / \mathrm{NPC}$ 非晶态合金电极在含有 $20 \mathrm{mmol} \cdot \mathrm{L}^{-1}$ 葡萄糖 1 $\mathrm{mol} \cdot \mathrm{L}^{-1} \mathrm{NaOH}$ 溶液中的不同电势扫描速率下的循环伏 安曲线. 从图 6A 中可以观察到, 随着扫描速率的增加, 峰电流也出现相应的增大, 氧化峰峰电位正移, 还原峰 峰电位负移. 在低的电势扫描速率下, 较难观察到还原 峰, 但随着电势扫描速率的增大, 还原峰可以明显观察 到. 这种现象表明 $\mathrm{Ni}(\mathrm{OH})_{2}$ 氧化为 $\mathrm{NiOOH}$ 的速率远远大

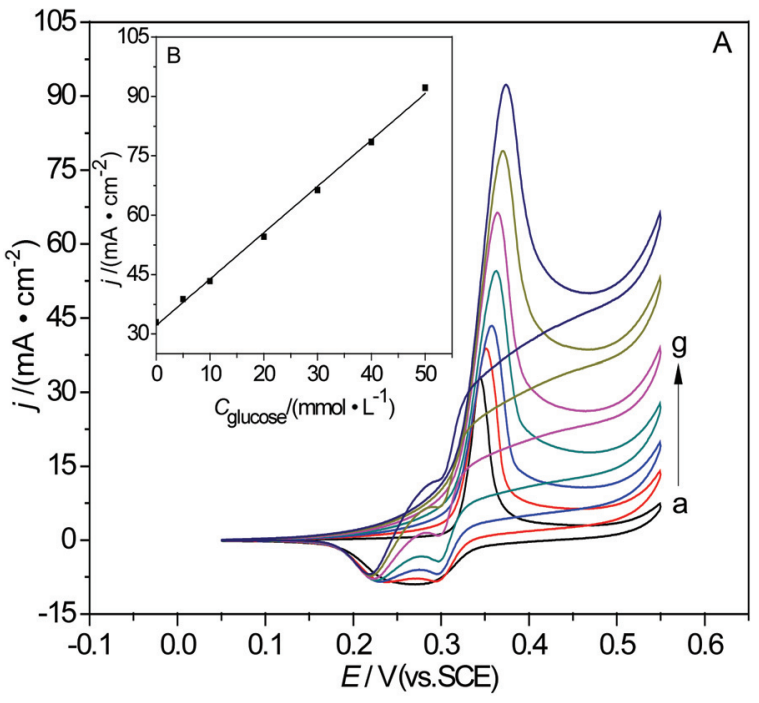

图 4 (A)高分散 Ni-B/NPC 非晶态合电极在含不同葡萄糖浓度的 1 $\mathrm{mol} \cdot \mathrm{L}^{-1} \mathrm{NaOH}$ 溶液中的循环伏安曲线. 扫速为 $10 \mathrm{mV} \cdot \mathrm{s}^{-1}$. 葡萄糖浓 度从 $\mathrm{a} \sim \mathrm{g}$ 依次为: $0,5,10,20,30,40$ 和 $50 \mathrm{mmol} \cdot \mathrm{L}^{-1}$. (B) 不同浓度葡萄 糖呈现的阳极峰电流密度 $(j)$ 与葡萄糖浓度的关系

Figure 4 (A) CV curves of the highly dispersed Ni-B/NPC amorphous alloy electrode in $1 \mathrm{~mol} \cdot \mathrm{L}^{-1} \mathrm{NaOH}$ solution in the presence of different concentrations of glucose at $10 \mathrm{mV} \cdot \mathrm{s}^{-1}$. Concentrations of glucose: 0,5 , $10,20,30,40$ and $50 \mathrm{mmol} \cdot \mathrm{L}^{-1}$ from a to $\mathrm{g}$. (B) The relationship between the anodic peak current and concentration of glucose

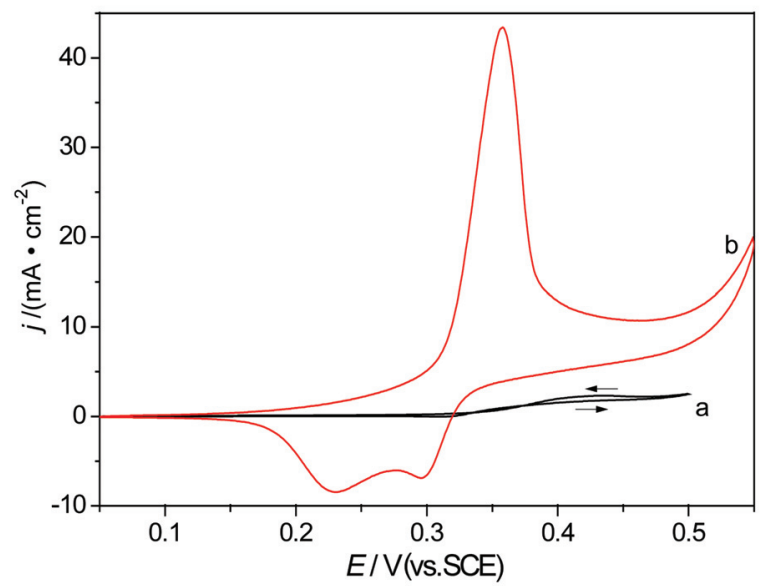

图 5 块状镍电极(a)和高分散 Ni-B/NPC 非晶态合金电极(b)在含 10 $\mathrm{mmol} \cdot \mathrm{L}^{-1}$ 葡萄糖的 $1 \mathrm{~mol} \cdot \mathrm{L}^{-1} \mathrm{NaOH}$ 溶液中的循环伏安曲线

Figure $5 \mathrm{CV}$ curves of the bulk nickel (a) and the highly dispersed $\mathrm{Ni}-\mathrm{B} / \mathrm{NPC}$ amorphous alloy (b) electrodes in $1 \mathrm{~mol} \cdot \mathrm{L}^{-1} \mathrm{NaOH}$ solution in the presence of $10 \mathrm{mmol} \cdot \mathrm{L}^{-1}$ glucose at $10 \mathrm{mV} \cdot \mathrm{s}^{-1}$

于葡萄糖电催化氧化的速率，同时揭露葡萄糖氧化在催 化剂表面可能是个缓慢的过程. 图 6B 为不同扫描速率 下呈现的葡萄糖阳极氧化峰电流 $\left(I_{\mathrm{p}, \mathrm{a}}\right)$ 与 $v^{1 / 2}$ 的关系. $I_{\mathrm{p}, \mathrm{a}}$ 与 $v^{1 / 2}$ 成良好的线性关系说明在 Ni-B/NPC 非晶态合金 电极上葡萄糖电催化氧化过程是受扩散控制的.

\section{4 高分散 Ni-B/NPC 非晶态合金电极在碱液中对葡 萄糖电催化氧化的动力学研究}

在研究电催化剂的催化性能中, 电子转移系数 $(\beta)$ 是一项重要的衡量参数, $\beta$ 越小, 反应的活化能越小, 说 


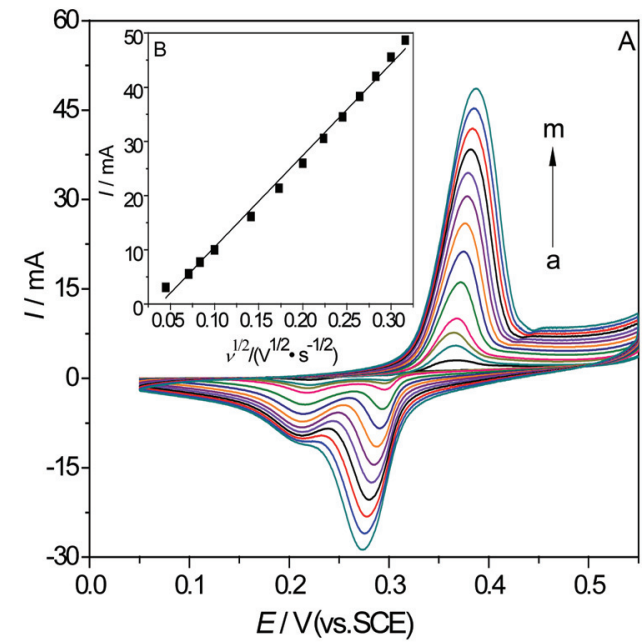

图 6 (A)高分散 $\mathrm{Ni}-\mathrm{B} / \mathrm{NPC}$ 非晶态合金电极在含 $20 \mathrm{mmol} \cdot \mathrm{L}^{-1}$ 葡萄糖 $1 \mathrm{~mol} \cdot \mathrm{L}^{-1} \mathrm{NaOH}$ 溶液中不同电势扫描速率下的循环伏安曲线. 从 $\mathrm{a} \sim$ $\mathrm{m}$ 依次为: $2,5,7,10,20,30,40,50,60,70,80,90$ 和 $100 \mathrm{mV} \cdot \mathrm{s}^{-1}$. (B) 不 同扫描速率下呈现的葡萄糖阳极氧化峰电流 $\left(I_{\mathrm{p}, \mathrm{a}}\right)$ 与扫描速率的平方根 $\left(v^{1 / 2}\right)$ 的关系

Figure 6 (A) $\mathrm{CV}$ curves of the highly dispersed Ni-B/NPC amorphous alloy electrode in $1 \mathrm{~mol} \cdot \mathrm{L}^{-1} \mathrm{NaOH}$ solution containing $20 \mathrm{mmol} \cdot \mathrm{L}^{-1}$ glucose at various potential sweep rate of $2,5,7,10,20,30,40,50,60$, $70,80,90$ and $100 \mathrm{mV} \cdot \mathrm{s}^{-1}$ from a to $\mathrm{m}$. (B) Dependence of the anodic peak current of glucose oxidation upon the square root of scan rate

\section{明反应越容易进行 ${ }^{[32]}$.}

图 7A 为高分散 Ni-B/NPC 非晶态合金电极在含 20 $\mathrm{mmol} \cdot \mathrm{L}^{-1}$ 葡萄糖 $1 \mathrm{~mol} \cdot \mathrm{L}^{-1} \mathrm{NaOH}$ 溶液中的稳态极化曲 线. 由文献 ${ }^{[33]}$ 可知葡萄糖氧化的速控步骤是单电子过 程, 可以通过葡萄糖氧化的稳态电位 $E$ 与 $\lg j$ 的关系曲 线计算电子转移系数 $\beta$. 图 $7 \mathrm{~B}$ 表示葡萄糖氧化反应的 Tafel 曲线, 其呈现 $\mathrm{S}$ 型, 说明在研究的电位范围内有不 同的葡萄糖氧化机理. 这是由于 $\beta-\mathrm{NiOOH}$ 和 $\gamma-\mathrm{NiOOH}$ 氧化葡萄糖所致. 因此，曲线被分成(1)和(2)两段，通过 $E$ 与 $\lg j$ 作图求得斜率, 对比 Tafel 公式 ${ }^{[12,34]}$ 计算出(1)和 (2)两阶段的电子转移系数 $\beta$ 分别为 0.62 和 0.88 . 说明 $\beta-\mathrm{NiOOH}$ 具有更强的活性, 是一种有效的氧化剂, 与文 献 $^{[31]}$ 报道的结果相吻合.

电催化反应速率常数是一项衡量电催化剂性能的 重要动力学参数. 双恒电位阶跃计时电流法是一种可用 于测定物质在电极上的电催化氧化速率常数的方法. 图 $8 \mathrm{~A}$ 为该电极在含不同浓度葡萄糖的 $1 \mathrm{~mol} \cdot \mathrm{L}^{-1} \mathrm{NaOH}$ 溶 液中双电位阶跃计时电流曲线. 由图 $8 \mathrm{~A}$ 可见, 在 400 $\mathrm{mV}$ 阶跃电位下, 响应电流随葡萄糖浓度的增加而增大, 且电流随时间增加很快趋于稳定, 表明该电极对葡萄糖 电氧化具有稳定的电催化活性.

巴德等 ${ }^{[35}$ 通过计时电流法提出电催化反应的机制 用于测定反应速率常数 $(k)$ :

$$
I_{\text {catal }} / I_{\mathrm{L}}=\lambda^{0.5}\left[\pi^{0.5} \operatorname{erf}\left(\lambda^{0.5}\right)+\exp (-\lambda) / \lambda^{0.5}\right]
$$

式中 $I_{\text {catal }}$ 和 $I_{\mathrm{L}}$ 分别表示含葡萄糖与空白溶液中的电流 值, $\lambda=k C t, C$ 为反应开始时葡萄糖的浓度 $\left(\mathrm{mol} \cdot \mathrm{cm}^{-3}\right) 、 t$

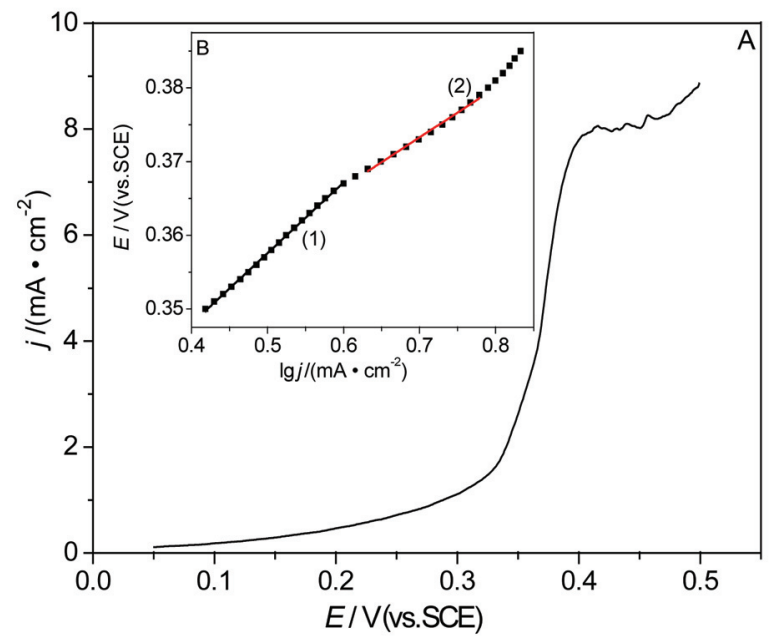

图 7 (A)高分散 $\mathrm{Ni}-\mathrm{B} / \mathrm{NPC}$ 非晶态合金电极在含 $20 \mathrm{mmol} \cdot \mathrm{L}^{-1}$ 葡萄糖 $1 \mathrm{~mol} \cdot \mathrm{L}^{-1} \mathrm{NaOH}$ 溶液中的稳态极化曲线. 扫速为 $0.1 \mathrm{mV} \cdot \mathrm{s}^{-1}$. (B) 高分 散 $\mathrm{Ni}-\mathrm{B} / \mathrm{NPC}$ 非晶态合金电极在含 $20 \mathrm{mmol} \cdot \mathrm{L}^{-1}$ 葡萄糖 $1 \mathrm{~mol} \cdot \mathrm{L}^{-1}$ $\mathrm{NaOH}$ 溶液中的 Tafel 曲线

Figure 7 (A) Steady polarization curve of the highly dispersed $\mathrm{Ni}-\mathrm{B} / \mathrm{NPC}$ amorphous alloy electrode in $1 \mathrm{~mol} \cdot \mathrm{L}^{-1} \mathrm{NaOH}$ solution containing $20 \mathrm{mmol} \cdot \mathrm{L}^{-1}$ glucose at $0.1 \mathrm{mV} \cdot \mathrm{s}^{-1}$. (B) Tafel plots for the highly dispersed Ni-B/NPC amorphous alloy electrode in $1 \mathrm{~mol} \cdot \mathrm{L}^{-1} \mathrm{NaOH}$ solution containing $20 \mathrm{mmol} \cdot \mathrm{L}^{-1}$ glucose

为反应时间(s). 在电催化反应中, $\lambda>1.5, \operatorname{erf}\left(\lambda^{0.5}\right) \rightarrow 1$, $\exp (-\lambda) / \lambda^{0.5} \rightarrow 0$, 式(1)近似化为:

$$
I_{\text {catal }} / I_{\mathrm{L}}=\lambda^{0.5} \pi^{0.5}=\pi^{0.5}(k C t)^{0.5}
$$

由图 $8 \mathrm{~B}$ 呈现 $I_{\text {catal }} / I_{\mathrm{L}}$ 与 $t^{1 / 2}$ 的关系曲线, 通过拟合直线斜 率，据式(2)，计算出葡萄糖在高分散 Ni-B/NPC 非晶态 合金电极上的电催化氧化反应速率常数 $k$ 为 $7.44 \times 10^{5}$ $\mathrm{cm}^{3} \cdot \mathrm{mol}^{-1} \cdot \mathrm{s}^{-1}$. 此值比 $\mathrm{Ni}-\mathrm{Cu} / \mathrm{GC}$ 电极得到的 $k\left(5.8 \times 10^{5}\right.$ $\mathrm{cm}^{3} \cdot \mathrm{mol}^{-1} \cdot \mathrm{s}^{-1}$ )大 1.28 倍 ${ }^{[14]}$.

双恒电位阶跃计时电流法也可以用来计算葡萄糖 的扩散系数 $(D)$, 由图 $8 \mathrm{C}$ 可知, 电流 $I$ 与 $t^{-1 / 2}$ 呈现良好 的线性关系，说明葡萄糖在 Ni-B 非晶态合金电极上的 电化学氧化反应是受扩散控制的. 根据 Cottrell 方程 ${ }^{[36]}$ :

$$
I=n F A D^{1 / 2} C \pi^{-1 / 2} t^{-1 / 2}
$$

式中 $n$ 是电极反应转移的电子数, $I$ 是响应电流值, $A$ 是 电极的表观面积. 通过 $I$ 对 $t^{-1 / 2}$ 作图的斜率, 再通过(3) 式可计算出葡萄糖的扩散系数 $(D)$ 为 $1.11 \times 10^{-3} \mathrm{~cm}^{2} \cdot \mathrm{s}^{-1}$. 此值比 $\mathrm{Ni}-\mathrm{Cu} / \mathrm{GC}$ 电极得到的 $D\left(1 \times 10^{-5} \mathrm{~cm}^{2} \cdot \mathrm{s}^{-1}\right)$ 大两个 数量级 ${ }^{[14]}$.

电化学交流阻抗是一种研究电极过程动力学的重 要方法. 图 9 呈现在含 $50 \mathrm{mmol} \cdot \mathrm{L}^{-1}$ 葡萄糖的 $1 \mathrm{~mol} \cdot \mathrm{L}^{-1}$ $\mathrm{NaOH}$ 溶液中高分散 $\mathrm{Ni}-\mathrm{B} / \mathrm{NPC}$ 非晶态合金电极和块 状镍电极的电化学 Nyquist 图和 Bode 图. 其相应的拟合 电路的等效电路如图 10 所示, 相应拟合得到的参数值 如表 2 所示. Nyquist 图的高频区和低频区可以观察到一 个挤压部分重叠的电容半圆弧. 高频区的电容半圆弧是 由 $\mathrm{NiOOH} / \mathrm{Ni}(\mathrm{OH})_{2}$ 相互转化的电荷传递电阻和双电层 电容并联造成的, 低频区的电容半圆是由反应中间产物 


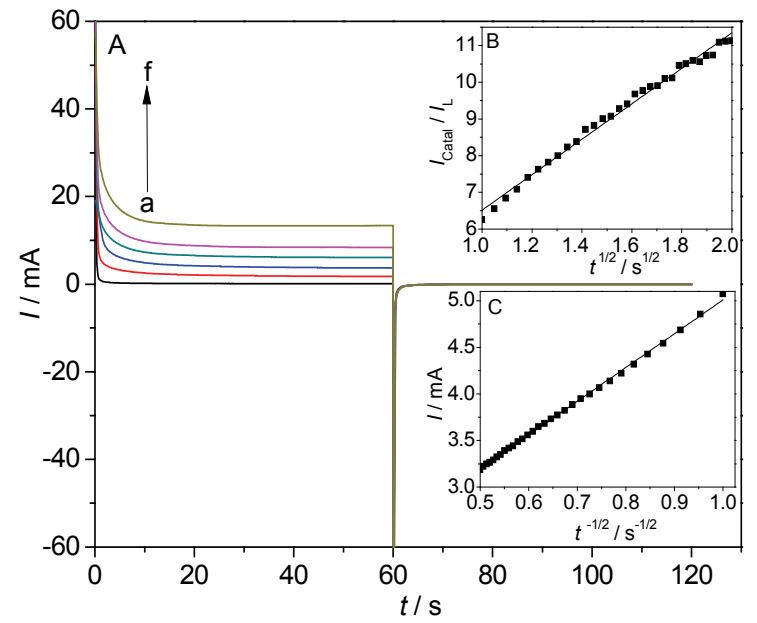

图 8 (A)为高分散 Ni-B/NPC 非晶态合金电极在含不同浓度葡萄糖 的 $1 \mathrm{~mol} \cdot \mathrm{L}^{-1} \mathrm{NaOH}$ 溶液中的双恒电位阶跃计时电流曲线. (B) 为含 10 $\mathrm{mmol} \cdot \mathrm{L}^{-1}$ 葡萄糖与不含葡萄糖的 $1 \mathrm{~mol} \cdot \mathrm{L}^{-1} \mathrm{NaOH}$ 溶液中呈现电流的 比值 $I_{\text {catal }} / I_{\mathrm{L}}$ 与 $t^{1 / 2}$ 的关系图. (C)为含 $10 \mathrm{mmol} \cdot \mathrm{L}^{-1}$ 葡萄糖的 $1 \mathrm{~mol} \cdot \mathrm{L}^{-1}$ $\mathrm{NaOH}$ 溶液呈现的响应电流扣除空白溶液响应电流后的电流 $I$ 与 $t^{-1 / 2}$ 关系图

Figure 8 (A) Double potential step chronoamperograms of the highly dispersed Ni-B/NPC amorphous alloy electrode in $1 \mathrm{~mol} \cdot \mathrm{L}^{-1} \mathrm{NaOH}$ solution with $0,10,20,30,40$ and $50 \mathrm{mmol} \cdot \mathrm{L}^{-1}$ glucose from a to $\mathrm{f}$. Step potentials were $400 \mathrm{mV}$ and $100 \mathrm{mV}$. (B) Plot of $I_{\text {catal }} / I_{\mathrm{L}}$ versus $t^{1 / 2}$ derived from the data of chronoamperograms in absence and in presence of 10 $\mathrm{mmol} \cdot \mathrm{L}^{-1}$ glucose in $1 \mathrm{~mol} \cdot \mathrm{L}^{-1} \mathrm{NaOH}$ solution. (C) Plot of $I$ versus $t^{-1 / 2}$ from the chronoamperogram in $1 \mathrm{~mol} \cdot \mathrm{L}^{-1} \mathrm{NaOH}$ solution containing 10 $\mathrm{mmol} \cdot \mathrm{L}^{-1}$ glucose after removing the background current

的吸附引起的 ${ }^{[20]}$. 由表 2 可知, 相比于块状镍电极, 高 分散 Ni-B/NPC 电极具有较低的电荷传递电阻和双电层 电容, 说明 Ni-B/NPC 电极具有更快的电子传递速率, 有利于葡萄糖氧化反应法拉第过程的发生 ${ }^{[37]}$, 从而降 低葡萄糖氧化起始电位. 该结果与 $\mathrm{CA}$ 和 $\mathrm{CV}$ 分析的结 果相吻合. 同时, Ni-B/NPC 电极具有较小的吸附电阻, 说明中间产物反应越充分, 电极催化反应速率越快. 进
一步说明葡萄糖在 Ni-B/NPC 电极上具有更高的电催化 氧化速率.

表 2 高分散 Ni-B/NPC 非晶态合金电极和块状镍电极上葡萄糖电氧 化反应等效电路的拟合参数值

Table 2 Fitted equivalent circuit parameters of electrooxidation of glucose at the highly dispersed Ni-B/NPC amorphous alloy and bulk $\mathrm{Ni}$ electrodes in $1 \mathrm{~mol} \cdot \mathrm{L}^{-1} \mathrm{NaOH}$ solution

\begin{tabular}{ccc}
\hline Category & \multicolumn{2}{c}{ Catalyst } \\
\cline { 2 - 3 } & Bulk Ni & Ni-B/NPC \\
\hline$R_{\mathrm{s}} /\left(\Omega \cdot \mathrm{cm}^{2}\right)$ & $0.42 \pm 0.03$ & $0.71 \pm 0.01$ \\
$C_{\mathrm{d} /} /\left(\mathrm{F} \cdot \mathrm{cm}^{-2}\right)$ & $(8.09 \pm 0.27) \times 10^{-5}$ & $0.012 \pm 0.0002$ \\
$R_{\mathrm{ct}} /\left(\Omega \cdot \mathrm{cm}^{2}\right)$ & $147.7 \pm 21.7$ & $40.7 \pm 1.6$ \\
$C_{\mathrm{ads}} /\left(\mathrm{F} \cdot \mathrm{cm}^{-2}\right)$ & $(1.16 \pm 0.15) \times 10^{-4}$ & $0.13 \pm 0.03$ \\
$R_{\mathrm{ads}} /\left(\Omega \cdot \mathrm{cm}^{2}\right)$ & $607.6 \pm 34.1$ & $18.83 \pm 1.9$ \\
\hline
\end{tabular}

\section{3 结论}

以纳米多孔铜为载体，采用超声辅助化学镀方法制 备出高分散 Ni-B/NPC 非晶态合金电极. CA 测试表明超 声辅助化学镀 $5 \mathrm{~min}$ 制备的高分散 Ni-B/NPC 非晶态合 金电极电化学活性表面积及粗䊁度最大. 与块状多晶镍 电极相比, CV 和 EIS 结果表明高分散 Ni-B/NPC 非晶态 合金电极对碱性介质中葡萄糖的电氧化具有较高的稳 定性和电催化活性. 此外, CA 计算结果表明葡萄糖在高 分散 $\mathrm{Ni}-\mathrm{B} / \mathrm{NPC}$ 非晶态合金电极上的 $k$ 值比 $\mathrm{Ni}-\mathrm{Cu} / \mathrm{GC}$ 电极得到的 $k$ 值大 1.28 倍, 葡萄糖的 $D$ 值比 $\mathrm{Ni}-\mathrm{Cu} / \mathrm{GC}$ 电极得到的 $D$ 大两个数量级. 其原因可能是 $\mathrm{Ni}-\mathrm{B} / \mathrm{NPC}$ 电极具有较大的电化学活性表面积, 可大大增加与电解 液的接触，使得表面吸附更多的葡萄糖分子，从而电势 正向扫描时形成较大的氧化电流. 该工作对研究直接葡 萄糖燃料电池非贵金属阳极催化剂具有一定的理论和 实践指导意义.
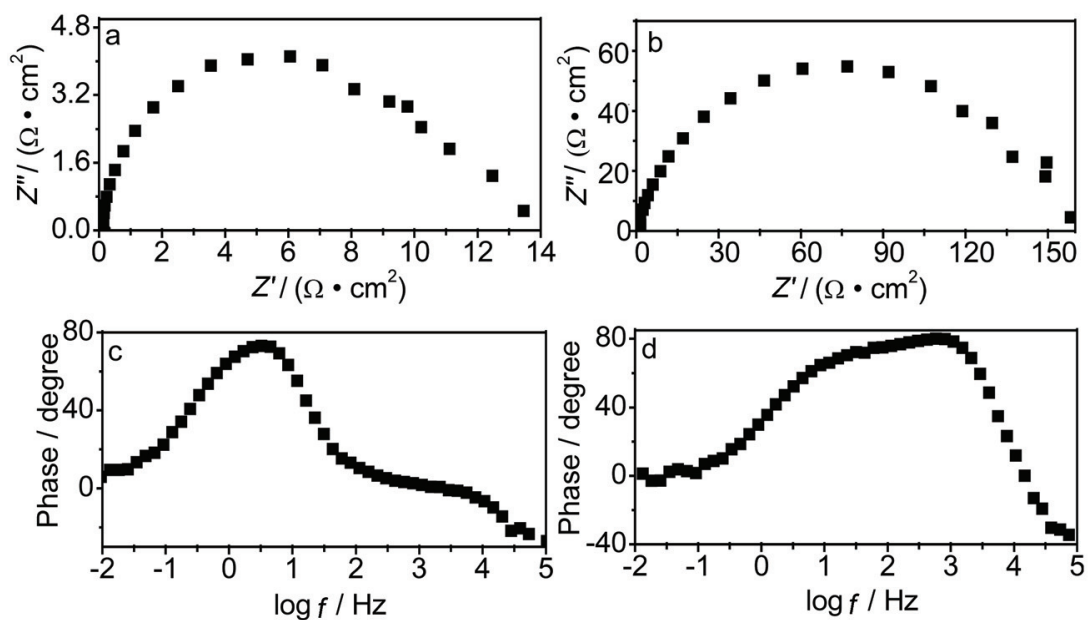

图 9 高分散 Ni-B/NPC 非晶态合金电极 $\left(\mathrm{a}\right.$ 和 $\mathrm{c}$ ) 和块状镍电极 $\left(\mathrm{b}\right.$ 和 $\mathrm{d}$ )在含 $50 \mathrm{mmol} \cdot \mathrm{L}^{-1}$ 葡萄糖 $1 \mathrm{~mol} \cdot \mathrm{L}^{-1} \mathrm{NaOH}$ 溶液中的 $\mathrm{Nyquist}$ 图和相图

Figure 9 Nyquist (a), Bode (c) plots of the highly dispersed Ni-B/NPC amorphous alloy electrode and Nyquist (b), Bode (d) plots of the bulk Ni electrode in $1 \mathrm{~mol} \cdot \mathrm{L}^{-1} \mathrm{NaOH}$ solution containing $50 \mathrm{mmol} \cdot \mathrm{L}^{-1}$ glucose. DC potential is $350 \mathrm{mV}$ 


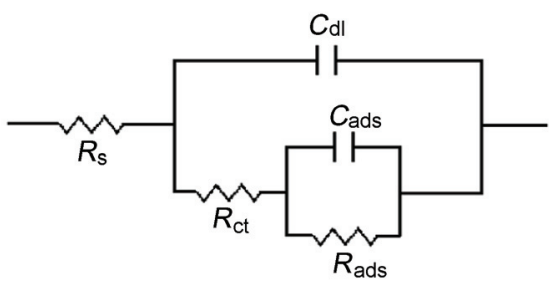

图 10 高分散 $\mathrm{Ni}-\mathrm{B} / \mathrm{NPC}$ 非晶态合金电极和块状镍电极上葡萄糖电氧 化反应等效电路图. $R_{\mathrm{s}}, C_{\mathrm{dl}}, R_{\mathrm{ct}}, C_{\mathrm{ads}}, R_{\mathrm{ads}}$ 分别代表溶液电阻, 双电层电 容, 电荷转移电阻, 吸附电容和吸附电阻

Figure 10 Electrical equivalent circuit diagram for modeling the Nyquist Plots of glucose electrooxidation at the highly dispersed Ni-B/NPC amorphous alloy and bulk Ni electrodes. $R_{\mathrm{s}}, C_{\mathrm{dl}}, R_{\mathrm{ct}}, C_{\mathrm{ads}}, R_{\mathrm{ads}}$ represent solution resistance, double layer capacitance, charge transfer resistance, electrical elements related to the adsorption of reaction intermediates and adsorption resistance

\section{4 实验部分}

用不同型号金相砂纸依次对 $0.2 \mathrm{~cm}^{2}$ 铜箔基底进行 打磨, 再依次进行超声波水洗、化学除油、水洗、化学 抛光、去离子水洗、 $10 \% \mathrm{HCl}$ 活化液中活化, 接着置于 镀锌液中, 用 ZF-9 恒电位/恒电流仪电沉积锌 $4 \mathrm{~min}$, 电 流密度为 $20 \mathrm{~mA} \cdot \mathrm{cm}^{-2}$. 然后将镀锌片放入真空干燥箱 中, 在 $\mathrm{N}_{2}$ 保护下 $180{ }^{\circ} \mathrm{C}$ 恒温热处理 $2 \mathrm{~h}$, 制备铜基表面 铜锌合金. 将合金放入 $30{ }^{\circ} \mathrm{C}$ 的质量分数为 $5 \% \mathrm{H}_{2} \mathrm{SO}_{4}$ 溶 液中进行去合金化处理 20,40,60 和 $120 \mathrm{~min}$, 溶解其中 的锌, 制备出纳米多孔铜材料. 腐蚀完后将纳米多孔铜 材料放入水中浸泡 $60 \mathrm{~min}$, 然后吹干备用. 以去合金化 处理 $60 \mathrm{~min}$ 制备的纳米多孔铜为基底, 在 $66{ }^{\circ} \mathrm{C}$ 的化学 镀液中通过超声辅助化学镀方法制备铜基高分散 Ni-B 非晶态合金电极. 反应系统置于超声波清洗器中, 超声 功率为 $60 \mathrm{~W}$, 分别制备化学镀 $3,5,10$ 和 $15 \mathrm{~min}$ 的 $\mathrm{Ni}-\mathrm{B} / \mathrm{NPC}$ 非晶态合金电极, 反应结束后将电极放入水 中浸泡 $60 \mathrm{~min}$, 最后吹干备用. $0.2 \mathrm{~cm}^{2}$ 块状镍簿片电极 依次用不同型号金相砂纸打磨至镜面光亮, 然后依次进 行超声波水洗、化学除油、水洗，最后吹干备用.

电化学测试在三电极体系中进行, 电解液分别为含 不同浓度葡萄糖的 $1 \mathrm{~mol} \cdot \mathrm{L}^{-1} \mathrm{NaOH}$ 溶液, 实验试剂均 为分析纯, 用去离子水配制, 系统置于 $25{ }^{\circ} \mathrm{C}$ 恒温水浴 中, 测试前通高纯 $\mathrm{N}_{2}$ 除氧 $15 \mathrm{~min}$. 电化学阻抗测试频 率范围为 $100 \mathrm{kHz} \sim 0.01 \mathrm{~Hz}$, 扰动电势幅值为 $10 \mathrm{mV}$, 偏压为 $350 \mathrm{mV}$. 电极测试前需经过 CV 扫描至前后两圈 重合以获得稳定的电极表面.

\section{References}

[1] Fujiwara, N.; Yamazaki, S. I.; Siroma, Z.; Ioroi, T.; Senoh, H.; Yasuda, K. Electrochem. Commun. 2009, 11, 390.

[2] Basu, D.; Basu, S. Electrochim. Acta 2010, 55, 5775.

[3] Basu, D.; Basu, S. Electrochim. Acta 2011, 56, 6106.

[4] Park, S.; Boo, H.; Chung, T. D. Anal. Chim. Acta 2006, 556, 46.
[5] Yang, Y. J.; Hu, S. S. Electrochim. Acta 2010, 55, 3471.

[6] Xu, Q.; Yin, L. N.; Hou, C. T.; Liu, X. X.; Hu, X. Y. Sens. Actuators $B$ 2012, 173, 716 .

[7] Wang, Q. Y.; Cui, X. Q.; Chen, J. L.; Zheng, X. L.; Liu, C.; Xue, T. Y.; Wang, H. T.; Jin, Z.; Qiao, L.; Zheng, W. T. RSC Adv. 2012, 2, 6245.

[8] Xia, Q. F.; Luo, D.; Li, Z. J. Acta Chim. Sinica 2012, 70, 2079. (夏 前芳, 罗丹, 李在均, 化学学报, 2012, 70, 2079.)

[9] Basu, D.; Basu, S. Int. J. Hydrogen Energy 2012, 37, 4678.

[10] Tao, B. R.; Miao, F. J.; Chu, P. K. Electrochim. Acta 2012, 65, 149.

[11] Basu, D.; Basu, S. Int. J. Hydrogen Energy 2011, 36, 14923.

[12] Yousef Elahi, M.; Heli, H.; Bathaie, S. Z.; Mousavi, M. F. J. Solid State Electrochem. 2007, 11, 273.

[13] Qiu, R.; Zhang, X. L.; Qiao, R.; Li, Y.; Kim, Y. I.; Kang, Y. S. Chem. Mater. 2007, 19, 4174.

[14] Jafarian, M.; Forouzandeh, F.; Danaee, I.; Gobal, F.; Mahjani, M. G. J. Solid State Electrochem. 2009, 13, 1171

[15] Zheng, L.; Zhang, J. Q.; Song, J. F. Electrochim. Acta 2009, 54, 4559 .

[16] Ganesh, V.; Farzana, S.; Berchmans, S. J. Power Sources 2011, 196, 9890.

[17] Chekin, F.; Bagheri, S.; Arof, A. K.; Abd Hamid, S. B. J Solid State Electrochem. 2012, 16, 3245.

[18] Chen, J. Y.; Zhao, C. X.; Zhi, M. M.; Wang, K. W.; Deng, L. L.; Xu, G. Electrochim. Acta 2012, 66, 133.

[19] Galindo, R.; Mazario, E.; Gutiérrez, S.; Morales, M. P.; Herrasti, P. J. Alloys Compd. 2012, 536S, S241.

[20] Danaee, I.; Jafarian, M.; Forouzandeh, F.; Gobal, F. Int. J. Chem. Kinet. 2012, 44, 712.

[21] El-Refaei, S. M.; Saleh, M. M.; Awad, M. I. J. Power Sources 2013, 223, 125.

[22] Erlebacher, J.; Aziz, M. J.; Karma, A.; Dimitrov, N.; Sieradzki, K. Nature 2001, 410, 450.

[23] Wu, Z. J.; Ge, S. H.; Zhang, M. H.; Li, W.; Mu, S. C.; Tao, K. Y. J. Phys. Chem. C 2007, 111, 8587.

[24] Han, Q.; Liu, K. R.; Chen, J. S.; Li, X.; Wei, X. J. Int. J. Hydrogen Energy 2004, 29, 243.

[25] Zheng, Y. X.; Yao, S. B.; Zhou, S. M. Electrochemistry 2007, 13, 307. (郑一雄, 姚士冰, 周绍民, 电化学, 2007, 13, 307.)

[26] Zheng, Y. X.; Yao, S. B.; Zhou, S. M. Acta Phys. -Chim. Sin. 2008, 24, 1643. (郑一雄, 姚士冰, 周绍民, 物理化学学报, 2008, 24, 1643.)

[27] Jia, F. L.; Yu, C. F.; Deng, K. J.; Zhang, L. Z. J. Phys. Chem. C 2007, 111, 8424.

[28] Shu, Y. D.; Chen, B. C. Metallurge Electrochemistry of Studing Methods, Certral South University of Technology Press, Changsha, 1990, pp. $177 \sim 178$. (舒余德, 陈白珍, 冶金电化学研究方法, 中 南工业大学出版社, 长沙, 1990, pp. 177 178.)

[29] Zhou, Z.; Yan, J.; Lin, J.; Zhang, Y. S. Chin. J. Power Sources 1999, 23, 319. (周震, 阎杰, 林进, 张允什, 电源技术, 1999, 23, 319.)

[30] Danaee, I.; Jafarian, M.; Forouzandeh, F.; Gobal, F. Int. J. Hydrogen Energy 2009, 34, 859.

[31] Tehrani, R. M. A.; Ab Ghani, S. Fuel Cells 2009, 9, 579.

[32] Laviron, E.; Roullier, L. J. Electroanal. Chem. 1980, 115, 65.

[33] Ojani, R.; Raoof, J. B.; Salmany-Afagh, P. J. Electroanal. Chem. 2004, 571, 1

[34] Shu,Y. D.; Chen, B. C. Metallurge Electrochemistry of Studing Methods, Certral South University of Technology Press, Changsha, 1990, p. 88. (舒余德, 陈白珍, 冶金电化学研究方法, 中南工业大 学出版社, 长沙, 1990, p. 88.)

[35] Bard, A. J.; Faulkner, L. R. Electrochemical Methods, Fundamentals and Applications, Chemical Industry Press, Beijing, 2005, pp. 329 352, translated by Shao, Y. H.; Zhu, G. Y.; Dong, X. D.; Zhang, B. L. (Bard, A. J.; Faulkner, L. R. 电化学方法原理和应用, 邵元华, 朱 果逸, 董献堆, 张柏林译, 化学工业出版社, 北京, 2005, pp. 329 352.)

[36] Bard, A. J.; Faulkner, L. R. Electrochemical Methods, Fundamentals and Applications, translated by Shao, Y. H.; Zhu, G. Y.; Dong, X. D.; Zhang, B. L. Chemical Industry Press, Beijing, 2005, p. 566. (Bard, A.J.; Faulkner, L. R. 电化学方法原理和应用, 邵元华, 朱果逸, 董献堆, 张柏林译, 化学工业出版社, 北京, 2005, p. 566.)

[37] Döner, A.; Telli, E.; Kardas, G. J. Power Sources 2012, 205, 71.

(Qin, X.) 